\title{
A stochastic approach to number of corona virus cases
}

\author{
H. ÜNÖZKAN, M. YILMAZ AND A.M. DERE
}

\begin{abstract}
This paper introduces a stochastic approach to case numbers of a pandemic disease. By defining the stochastic process random walk process is used. Some stochastic aspects for this disease are argued before stochastic study is started. During random walk process modeling new patients, recovering patients and dead conclusions are modelled and probabilities changes in some stages. Let the structure of this study includes vanishing process as a walk step, some wave happenings like big differences about spread speed as a big step in treatment- an effective vaccine or an influential chemical usage- a second corona virus pumping with virus mutation, a second global happening which bumping virus spread are defined as stages. This study only simulates a stochastic process of corona virus effects.
\end{abstract}

Mathematics Subject Classification: 60J74

Keywords: Random walk; vanishing probability; stochastic process; stationary probability; Markov chain.

\section{INTRODUCTION}

In industrial engineering, studying in reliability and stochastic processes of systems is very popular. In many situations this issue has vital importance. Besides there are many studies with random walks and many of them include independent increases. In a study a new class is determined by phase type distribution and in [1] bivariate geometric distribution was chosen as a discrete phase type.

Let $X_{1}, X_{2}, \ldots, X_{N}$ be a sequence of independent and identically distributed (iid) random variables independent of the discrete random variable $N$. Commonly, the main issue is to determine the distribution of the compound random variable $S\left(X_{1}, X_{2}, \ldots, X_{N}\right)$ for popular choices of $\mathrm{S}$, to illustrate $S\left(x_{1}, x_{2}, \ldots, x_{N}\right)=\sum_{i=1}^{n} x_{i}$, $S\left(x_{1}, x_{2}, \ldots, x_{N}\right)=\min \left(x_{1}, x_{2}, \ldots, x_{N}\right), \quad S\left(x_{1}, x_{2}, \ldots, x_{N}\right)=\min \left(x_{1}, x_{2}, \ldots, x_{N}\right)$. Some studies in this area are in [2]-[5] .

The discrete phase type distributions are quite rich in modeling waiting time distributions like geometric, negative binomial, and bivariate geometric distribution. 
In a Markov process a definition for discrete phase type distribution can be defined with $d$ transient states and an absorbing state may be defined as " 0 ".

In [6] extreme shock models in phase type was studied and by this optimal component changes determined. At first rules in shock models assumed and later optimization calculated with phase type distribution.

In [7], multivariate Poisson process combined with Markov process by usability of phase type in shock models.

In this study a stochastic approach is introduced and the process of this system is studied. This random walk system includes vanishing process for dead and recovered patients, new patients and the probabilities in probability transition are changing with some stages that define big differences about spread speed as a big step in treatment- an effective vaccine or an influential chemical usage- a second corona virus wave with virus mutation and a second global happening which bumps virus spread.

\section{MATERIAL AND METHODS}

\subsection{Multivariate geometric distribution}

The univariate geometric distribution is famous to be the sole discrete distribution which has memoryless property. Multivariate geometric distribution has been studied in many articles [8]-[12]. Especially in shock models this memoryless specialty is studied commonly.

Every multivariate extension in geometric distribution have the same memoryless specialty and there can be widespread seen on studies about MarshallOlkin multivariate exponential distribution via multivariate geometric compounding of exponentially distributed random variables.

Bivariate geometric distribution was examined in a study and the formulation is as follow [13].

$\operatorname{Pr}(N=n, M=m)=\sum \sum\left(\begin{array}{c}m+n \\ n\end{array}\right) p_{1}{ }^{n} p_{2}{ }^{m} p_{0} \quad p_{0}+p_{1}+p_{2}=1 \mathrm{n}, \mathrm{m}=0,1,2, \ldots$ $p_{0}$ : probability of no adding, $p_{1}$ : probability of adding to upper part, 
$p_{2}$ : probability of adding to lower part.

To decide the number of new components in system marginal distribution functions are needed. We reach marginal distributions as follow.

$\operatorname{Pr}(N=n)=\sum_{m=0}^{\infty} \frac{(m+n) !}{m ! n !} p_{1}{ }^{n} p_{2}{ }^{m} p_{0}=\frac{p_{1}{ }^{n} p_{0}}{\left(1-p_{2}\right)^{n+1}} \sum_{m=0}^{\infty} \frac{(m+n) !}{m ! n !} p_{2}{ }^{m}\left(1-p_{2}\right)^{n+1}$

With using negative binomial distribution the sum is 1 . So the first marginal distribution is in equation (1).

$$
\operatorname{Pr}(N=n)=\frac{p_{1}{ }^{n} p_{0}}{\left(1-p_{2}\right)^{n+1}}
$$

Geometric distribution with success rate $\frac{p_{0}}{p_{0}+p_{1}}$ is gained. Second marginal distribution can be obtained with the same way in equation (2).

$$
\operatorname{Pr}(M=m)=\frac{p_{2}{ }^{m} p_{0}}{\left(1-p_{1}\right)^{m+1}}
$$

Therefore geometric distribution with $\frac{p_{0}}{p_{0}+p_{2}}$ success rate is gained.

In [14] applications of reliabilities in bivariate geometric distribution was assessed and gained characteristics of this valuable distribution.

In that study moment generating function and characteristics obtained as below.

$$
\begin{aligned}
& E\left(e^{t_{1} n+t_{2} m}\right)=\sum_{n=1}^{\infty} \sum_{m=1}^{\infty} e^{t_{1} n+t_{2} m}\left(\begin{array}{c}
m+n \\
n
\end{array}\right)\left(p_{1}\right)^{n}\left(p_{2}\right)^{m} p_{3} \\
& =\frac{p_{3}}{1-p_{1} e^{t_{1}}-p_{2} e^{t_{2}}} \sum_{n=1}^{\infty} \sum_{m=1}^{\infty}\left(\begin{array}{c}
m+n \\
n
\end{array}\right)\left(e^{t_{1}} p_{1}\right)^{n}\left(e^{t_{2}} p_{2}\right)^{m}\left(1-p_{1} e^{t_{1}}-p_{2} e^{t_{2}}\right) \\
& =\frac{p_{3}}{1-p_{1} e^{t_{1}}-p_{2} e^{t_{2}}} \\
& E(N)=\left.\frac{d}{d t_{1}}\left(\frac{p_{3}}{1-p_{1} e^{t_{1}}-p_{2} e^{t_{2}}}\right)\right|_{t_{1}=0, t_{2}=0}=\frac{p_{3} p_{1}}{\left(1-p_{1}-p_{2}\right)^{2}}=\frac{p_{1}}{p_{3}} \\
& E(M)=\frac{p_{2}}{p_{3}} \\
& E(N M)=\left.\frac{d^{2}}{d t_{1} d t_{2}}\left(\frac{p_{3}}{1-p_{1} e^{t_{1}}-p_{2} e^{t_{2}}}\right)\right|_{t_{1}=0, t_{2}=0}=\frac{2 p_{1} p_{3}}{p_{3}^{2}} \\
& E\left(N^{2}\right)=\left.\frac{d^{2}}{d t_{1}{ }^{2}}\left(\frac{p_{3}}{1-p_{1} e^{t_{1}}-p_{2} e^{t_{2}}}\right)\right|_{t_{1}=0, t_{2}=0}=\frac{p_{1}}{p_{3}}\left(1+\frac{2 p_{1}}{p_{3}}\right)
\end{aligned}
$$




$$
E\left(M^{2}\right)=\left.\frac{d^{2}}{d t_{2}{ }^{2}}\left(\frac{p_{3}}{1-p_{1} e^{t_{1}}-p_{2} e^{t_{2}}}\right)\right|_{t_{1}=0, t_{2}=0}=\frac{p_{2}}{p_{3}}\left(1+\frac{2 p_{2}}{p_{3}}\right)
$$

\subsection{Markov chain}

When the next position of a system rely solely on its current position and not on its prior positions, the system mostly be assumed to be a Markovian process. A kind of this model is suggested in [15]. In that process, Markov model was used for describing deterioration.

We pay attention to Markov process by defining a finite state space with either a discrete or continuous time parameter [16]. In literature there have been many studies in which a more general process called a "semi-Markov process" was discussed. This process includes discrete time parameter Markov chain. Some processes in semi-Markov process may be used to describe some classes of systems related to inspection, replacement, and repair.

When we try to find a definite form of steps with using bivariate geometric transition probabilities;

$$
P_{i j}^{n}=P\left(X_{n+1}=j \mid X_{n}=i\right)
$$

We may assume transition probabilities are independent.

When the comings are independent, then there are two different marginal geometric distributions which are available for comings. Their probability values are $\frac{p_{0}}{p_{0}+p_{1}}$ and $\frac{p_{0}}{p_{0}+p_{2}}$.

When the arrivals are dependent, then first marginal distribution is geometric distribution with probability value $\frac{p_{0}}{p_{0}+p_{1}}$ and the other marginal distribution is negative binomial distribution with unsuccessful try number $m$, success number is $n$. 


\subsection{Vanishing process}

Assume that the time till vanishing of a Markov sequence which has finite state space is shown with random variable $T$.

Random variable $T$ has a discrete phase type distribution and probability function is as below,

$$
P(T=n)=\underline{a} Q^{n-1} \underline{t}^{\prime}
$$

$Q$ is a matrix which includes transition probabilities between transition states,

$\underline{t}$ is a vector which includes transition probabilities between transition states to vanishing state

$\underline{a}$ is a sub vector of starting probabilities.

\section{Example (Geometric Distribution)}

Describing trial numbers till first success. $X_{n}$ shows the value in $n^{\text {th }}$ try. So $X_{n} \in$ $\{0,1\}, 1$ is vanishing state. The probability transition matrix is as follows,

$$
p=\begin{aligned}
& 0 \\
& 1
\end{aligned} \quad \begin{array}{cc}
1-p & p \\
0 & 1
\end{array} \|
$$

$Q=1-p, \underline{t^{\prime}}=p, \underline{a}=1$

$$
P(T=n)=(1-p)^{n-1} p
$$

Thus we may say discrete phase type distributions is a generalized version of geometric distribution.

\subsection{Random walk process}

Assume $\left\{Y_{n}, n=1,2, \ldots\right\}$ is a sequence of random variables with d-dimensional and it is independently identical distributed. $X_{0}$ is a constant vector in $\mathbb{R}^{d}$.

$\left\{X_{n}, n=1,2, \ldots\right\}$ process which is defined with $X_{n}=X_{0}+Y_{1}+\cdots+Y_{n}, n=1,2, \ldots$ is called

d-dimensional random walk. If $X_{0}$ and $Y_{n}$ valued in $\mathbb{Z}^{d}$ space than $\left\{X_{n}, n=1,2, \ldots\right\}$ process is called d-dimensional cage random walk. In cage random walk when $\varepsilon_{k}=$ -1 or $1, k=1.2 \ldots, d$ and $Y_{n}$ jumps only from $x=\left(x_{1}, x_{2}, \ldots, x_{d}\right)$ to $y=$ $\left(x_{1}+\varepsilon_{1}, x_{2}+\varepsilon_{2}, \ldots, x_{d}+\varepsilon_{d}\right)$ than this is called simple random walk. 
For a simple random walk for any given jump each jumps from $2 d$ moves happens with probability $p=\frac{1}{2 d}$ thereby this process is called symmetrical random walk process.

In all these situations when $Y_{n}$ jumps are only independent but not with identical distribution than $\left\{X_{n}, n=1,2, \ldots\right\}$ process is called non-homogenous random walk.

$$
\begin{gathered}
X_{1}=X_{0}+Y_{1} \\
X_{2}=X_{1}+Y_{2} \\
\cdot \\
\cdot \\
\cdot \\
X_{n}=X_{n-1}+Y_{n}, n=1,2, \ldots
\end{gathered}
$$

Thus a random walk is independently incremental and stationary incremental.

\section{RESULTS AND DISCUSSIONS}

The structure of our approach includes two different options which are increasing and vanishing processes. When the next step goes to increasing the number of patients increases with new additions, on the other hand when the next step goes to vanishing process the number of patients decreases with dead or recover. Our approach assumes some steps which define the rules of this complex structure.

The process starts after 100000 patients determined.

- $\quad$ The increasing probability in transition is $p$, and vanishing probability in transition is $q$.

$X_{n}$ shows the conclusion of corona disease after $n$ step.

In the first 15 days we assume $p=3 q$.

- $\quad$ In the second 15 days we assume $p=q$. Because some precautions may take into account.

- $\quad$ In the third 15 days with more strict precautions $p=\frac{1}{3} q$.

- $\quad$ In the fourth 15 days people may be pay less attention to disease $p=q$.

After 60 days we assume the probabilities as $p=\frac{1}{3} q$ till the disease is over. 
$p_{0}$ represents no change in both ways - increasing patient number and vanishing patients. $p_{0}=0.001$ in every step.

- When no change in both ways occurs, the system halts.

- When a walk goes to increases jump it adds $\% 5$ of patient number in the starting point of step adding to the patient number and when a walk goes to decreases jump it goes $\% 5$ of patient number in the starting point to reduce down with recover or dead patients.

\section{APPLICATION}

In every step there are formed many equations that they create path of the process. Some examples are as follows;

$$
\begin{gathered}
P\left(X_{2}=0, X_{4}=0\right)=P\left(X_{2}=0\right) P\left(X_{4}-X_{2}=0\right)=P\left(X_{2}=0\right) P\left(X_{2}=0\right) \\
P\left(X_{4}=2, X_{10}=6\right)=P\left(X_{4}=2\right) P\left(X_{10}-X_{4}=4\right)=P\left(X_{4}=2\right) P\left(X_{6}=4\right) \\
P\left(X_{4}=2, X_{10}=6, X_{14}=8\right)=P\left(X_{4}=2\right) P\left(X_{10}-X_{4}=4\right) P\left(X_{14}-X_{10}=2\right) \\
=P\left(X_{4}=2\right) P\left(X_{6}=4\right) P\left(X_{4}=2\right)
\end{gathered}
$$

Expectation numbers of increases and decreases in the path of the first step process may be find as below;

$$
E(N)=\left.\frac{d}{d t_{1}}\left(\frac{p_{3}}{1-p_{1} e^{t_{1}}-p_{2} e^{t_{2}}}\right)\right|_{t_{1}=0, t_{2}=0}=\frac{p_{3} p_{1}}{\left(1-p_{1}-p_{2}\right)^{2}}=\frac{p_{1}}{p_{3}}
$$

After gaining this expectation we consider that whether this walk does not stop in 15 step, we expect that this walk includes $E(N)$ increases and we will find the other expectation for decreases with

$$
E(M)=\left.\frac{d}{d t_{2}}\left(\frac{p_{3}}{1-p_{1} e^{t_{1}}-p_{2} e^{t_{2}}}\right)\right|_{t_{1}=0, t_{2}=0}=\frac{p_{3} p_{2}}{\left(1-p_{1}-p_{2}\right)^{2}}=\frac{p_{2}}{p_{3}}
$$

And we portion the expectations for gaining 15 step expectations.

$$
\begin{aligned}
& \text { expectation of increases }=15 \frac{E(N)}{E(M)+E(N)} \\
& \text { expectation of decreases }=15 \frac{E(M)}{E(M)+E(N)}
\end{aligned}
$$


Every jump position in random walk only relates to previous one not the others so every jump has basic Markov process specialty. Some of the transition probability matrices of the first step of this system are as below. These matrices tell us the jumps' position probability from the beginning.

$$
\begin{gathered}
P=\left[\begin{array}{ccc}
1 & 0 & 0 \\
0.001 & 0.74925 & 0.24975 \\
0.001 & 0.74925 & 0.24975
\end{array}\right] \\
P^{2}=\left[\begin{array}{ccc}
1 & 0 & 0 \\
0.002 & 0.7485 & 0.2495 \\
0.002 & 0.7485 & 0.2495
\end{array}\right] \\
P^{3}=\left[\begin{array}{ccc}
1 & 0 & 0 \\
0.004 & 0.747 & 0.249 \\
0.004 & 0.747 & 0.249
\end{array}\right]
\end{gathered}
$$

There may be many different paths of the same position in the same step. So each path must be included in the calculation of the expectation.

$$
E\left(X_{n}\right)=\sum_{i=1}^{n} P\left(X_{i}=x_{i}\right) x_{i}
$$

To illustrate, a graph of a path is in figure 1.

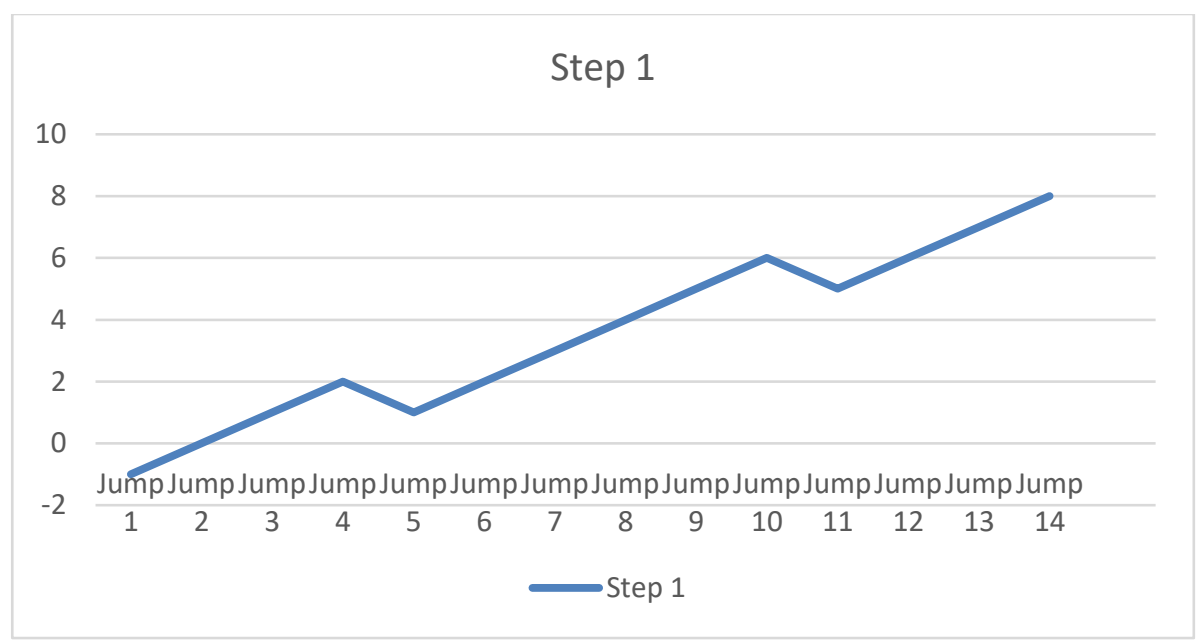

Fig. 1. One path of step 1 


\section{Step 1}

Some of probability transition matrices are as below;

$$
\begin{aligned}
P & =\left[\begin{array}{ccc}
1 & 0 & 0 \\
0.001 & 0.74925 & 0.24975 \\
0.001 & 0.74925 & 0.24975
\end{array}\right] \\
P^{2} & =\left[\begin{array}{ccc}
1 & 0 & 0 \\
0.002 & 0.7485 & 0.2495 \\
0.002 & 0.7485 & 0.2495
\end{array}\right] \\
P^{3} & =\left[\begin{array}{ccc}
1 & 0 & 0 \\
0.004 & 0.747 & 0.249 \\
0.004 & 0.747 & 0.249
\end{array}\right]
\end{aligned}
$$

Some of paths and probability calculations are as follow;

$$
\begin{gathered}
P\left(X_{2}=0, X_{4}=0\right)=P\left(X_{2}=0\right) P\left(X_{4}-X_{2}=0\right)=P\left(X_{2}=0\right) P\left(X_{2}=0\right) \\
=P\left(X_{2}=0\right)^{2}=\left(P\left(X_{1}=-1\right), P\left(X_{2}=0\right)+P\left(X_{1}=1\right), P\left(X_{2}=0\right)\right)^{2} \\
=\left(P\left(X_{1}=-1\right), P\left(X_{2}=0\right)+P\left(X_{1}=1\right), P\left(X_{2}=0\right)\right)^{2} \\
=\left(P\left(X_{1}=-1\right) P\left(X_{1}=1\right)+P\left(X_{1}=1\right) P\left(X_{1}=-1\right)\right)^{2} \\
=(0.74925 * 0.24975+0.24975 * 0.74925)^{2}=0.14 \\
=4\left(X_{4}=2, X_{10}=6\right)=P\left(X_{4}=2\right) P\left(X_{10}-X_{4}=4\right)=P\left(X_{4}=2\right) P\left(X_{6}=4\right) \\
=4 P\left(X_{1}=-1\right) P\left(X_{1}=1\right)^{3} 6 P\left(X_{1}=-1\right) P\left(X_{1}=1\right)^{5}=0.42 * 0.3538=0.1486
\end{gathered}
$$

The probabilities of each situation with including every path is in Table 1 .

$$
\begin{gathered}
E\left(X_{n}\right)=\sum_{i=1}^{n} P\left(X_{i}=x_{i}\right) x_{i} \\
E\left(X_{15}\right)=P\left(X_{1}=1\right)^{15} P\left(X_{1}=-1\right)^{0} *(100000 *(1+15 * 0.05-0 * 0.005)) \\
+P\left(X_{1}=1\right)^{14} P\left(X_{1}=-1\right)^{1} *(100000 *(1+14 * 0.05-1 * 0.005)) \\
\cdot \\
\cdot \\
\cdot P\left(X_{1}=1\right)^{0} P\left(X_{1}=-1\right)^{15} *(100000 *(1+0 * 0.05-15 * 0.005)) \\
=135451
\end{gathered}
$$




\section{Step 2}

Some of probability transition matrices in the second 15 days are as below;

$$
\begin{aligned}
P & =\left[\begin{array}{ccc}
1 & 0 & 0 \\
0.001 & 0.4995 & 0.4995 \\
0.001 & 0.4995 & 0.4995
\end{array}\right] \\
P^{2} & =\left[\begin{array}{ccc}
1 & 0 & 0 \\
0.002 & 0.499 & 0.499 \\
0.002 & 0.499 & 0.499
\end{array}\right] \\
P^{3} & =\left[\begin{array}{ccc}
1 & 0 & 0 \\
0.004 & 0.498 & 0.498 \\
0.004 & 0.498 & 0.498
\end{array}\right]
\end{aligned}
$$

Some of paths and probability calculations are as follow;

$$
\begin{gathered}
P\left(X_{2}=0, X_{4}=0\right)=P\left(X_{2}=0\right) P\left(X_{4}-X_{2}=0\right)=P\left(X_{2}=0\right) P\left(X_{2}=0\right) \\
=P\left(X_{2}=0\right)^{2}=\left(P\left(X_{1}=-1\right), P\left(X_{2}=0\right)+P\left(X_{1}=1\right), P\left(X_{2}=0\right)\right)^{2} \\
=\left(P\left(X_{1}=-1\right), P\left(X_{2}=0\right)+P\left(X_{1}=1\right), P\left(X_{2}=0\right)\right)^{2} \\
=\left(P\left(X_{1}=-1\right) P\left(X_{1}=1\right)+P\left(X_{1}=1\right) P\left(X_{1}=-1\right)\right)^{2} \\
=(0.4995 * 0.4995+0.4995 * 0.4995)^{2}=0.249 \\
P\left(X_{4}=2, X_{10}=6\right)=P\left(X_{4}=2\right) P\left(X_{10}-X_{4}=4\right)=P\left(X_{4}=2\right) P\left(X_{6}=4\right) \\
=4 P\left(X_{1}=-1\right) P\left(X_{1}=1\right)^{3} 6 P\left(X_{1}=-1\right) P\left(X_{1}=1\right)^{5}=0.249 * 0.0931 \\
=0.3421
\end{gathered}
$$

The probabilities of each situation with including every path is in Table 1 .

$$
\begin{gathered}
E\left(X_{n}\right)=\sum_{i=1}^{n} P\left(X_{i}=x_{i}\right) x_{i} \\
E\left(X_{15}\right)=P\left(X_{1}=1\right)^{15} P\left(X_{1}=-1\right)^{0} *(135451 *(1+15 * 0.05-0 * 0.005)) \\
+P\left(X_{1}=1\right)^{14} P\left(X_{1}=-1\right)^{1} *(135451 *(1+14 * 0.05-1 * 0.005)) \\
\cdot \\
\cdot \\
\cdot P\left(X_{1}=1\right)^{0} P\left(X_{1}=-1\right)^{15} *(135451 *(1+0 * 0.05-15 * 0.005)) \\
=133433
\end{gathered}
$$




\section{Step 3}

Some of probability transition matrices are as below;

$$
\begin{aligned}
P & =\left[\begin{array}{ccc}
1 & 0 & 0 \\
0.001 & 0.24975 & 0.74925 \\
0.001 & 0.24975 & 0.74925
\end{array}\right] \\
P^{2} & =\left[\begin{array}{ccc}
1 & 0 & 0 \\
0.002 & 0.2495 & 0.7485 \\
0.002 & 0.2495 & 0.7485
\end{array}\right] \\
P^{3} & =\left[\begin{array}{ccc}
1 & 0 & 0 \\
0.004 & 0.249 & 0.747 \\
0.004 & 0.249 & 0.747
\end{array}\right]
\end{aligned}
$$

Some of paths and probability calculations are as follow;

$$
\begin{gathered}
P\left(X_{2}=0, X_{4}=0\right)=P\left(X_{2}=0\right) P\left(X_{4}-X_{2}=0\right)=P\left(X_{2}=0\right) P\left(X_{2}=0\right) \\
=P\left(X_{2}=0\right)^{2}=\left(P\left(X_{1}=-1\right), P\left(X_{2}=0\right)+P\left(X_{1}=1\right), P\left(X_{2}=0\right)\right)^{2} \\
=\left(P\left(X_{1}=-1\right), P\left(X_{2}=0\right)+P\left(X_{1}=1\right), P\left(X_{2}=0\right)\right)^{2} \\
=\left(P\left(X_{1}=-1\right) P\left(X_{1}=1\right)+P\left(X_{1}=1\right) P\left(X_{1}=-1\right)\right)^{2} \\
=(0.74925 * 0.24975+0.24975 * 0.74925)^{2}=0.14 \\
P\left(X_{4}=2, X_{10}=6\right)=P\left(X_{4}=2\right) P\left(X_{10}-X_{4}=4\right)=P\left(X_{4}=2\right) P\left(X_{6}=4\right) \\
=4 P\left(X_{1}=-1\right) P\left(X_{1}=1\right)^{3} 6 P\left(X_{1}=-1\right) P\left(X_{1}=1\right)^{5}=0.0155 * 0.0043 \\
=0.0198
\end{gathered}
$$

The probabilities of each situation with including every path is in Table 1 .

$$
\begin{gathered}
E\left(X_{n}\right)=\sum_{i=1}^{n} P\left(X_{i}=x_{i}\right) x_{i} \\
E\left(X_{15}\right)=P\left(X_{1}=1\right)^{15} P\left(X_{1}=-1\right)^{0} *(133433 *(1+15 * 0.05-0 * 0.005)) \\
+P\left(X_{1}=1\right)^{14} P\left(X_{1}=-1\right)^{1} *(133433 *(1+14 * 0.05-1 * 0.005)) \\
\cdot \\
\cdot \\
\cdot \\
+P\left(X_{1}=1\right)^{0} P\left(X_{1}=-1\right)^{15} *(133433 *(1+0 * 0.05-15 * 0.005)) \\
=82153
\end{gathered}
$$




\section{Step 4}

Some of probability transition matrices in the fourth 15 days are as below;

$$
\begin{aligned}
P & =\left[\begin{array}{ccc}
1 & 0 & 0 \\
0.001 & 0.4995 & 0.4995 \\
0.001 & 0.4995 & 0.4995
\end{array}\right] \\
P^{2} & =\left[\begin{array}{ccc}
1 & 0 & 0 \\
0.002 & 0.499 & 0.499 \\
0.002 & 0.499 & 0.499
\end{array}\right] \\
P^{3} & =\left[\begin{array}{ccc}
1 & 0 & 0 \\
0.004 & 0.498 & 0.498 \\
0.004 & 0.498 & 0.498
\end{array}\right]
\end{aligned}
$$

Some of paths and probability calculations are as follow;

$$
\begin{gathered}
P\left(X_{2}=0, X_{4}=0\right)=P\left(X_{2}=0\right) P\left(X_{4}-X_{2}=0\right)=P\left(X_{2}=0\right) P\left(X_{2}=0\right) \\
=P\left(X_{2}=0\right)^{2}=\left(P\left(X_{1}=-1\right), P\left(X_{2}=0\right)+P\left(X_{1}=1\right), P\left(X_{2}=0\right)\right)^{2} \\
=\left(P\left(X_{1}=-1\right), P\left(X_{2}=0\right)+P\left(X_{1}=1\right), P\left(X_{2}=0\right)\right)^{2} \\
=\left(P\left(X_{1}=-1\right) P\left(X_{1}=1\right)+P\left(X_{1}=1\right) P\left(X_{1}=-1\right)\right)^{2} \\
=(0.4995 * 0.4995+0.4995 * 0.4995)^{2}=0.249 \\
P\left(X_{4}=2, X_{10}=6\right)=P\left(X_{4}=2\right) P\left(X_{10}-X_{4}=4\right)=P\left(X_{4}=2\right) P\left(X_{6}=4\right) \\
=4 P\left(X_{1}=-1\right) P\left(X_{1}=1\right)^{3} 6 P\left(X_{1}=-1\right) P\left(X_{1}=1\right)^{5}=0.249 * 0.0931 \\
=0.3421
\end{gathered}
$$

The probabilities of each situations with including every path is in Table 1 .

$$
\begin{gathered}
E\left(X_{n}\right)=\sum_{i=1}^{n} P\left(X_{i}=x_{i}\right) x_{i} \\
E\left(X_{15}\right)=P\left(X_{1}=1\right)^{15} P\left(X_{1}=-1\right)^{0} *(82153 *(1+15 * 0.05-0 * 0.005)) \\
+P\left(X_{1}=1\right)^{14} P\left(X_{1}=-1\right)^{1} *(82153 *(1+14 * 0.05-1 * 0.005)) \\
\cdot \\
\cdot \\
\cdot \\
+P\left(X_{1}=1\right)^{0} P\left(X_{1}=-1\right)^{15} *(82153 *(1+0 * 0.05-15 * 0.005)) \\
=80929
\end{gathered}
$$




\section{Step 5}

Some of probability transition matrices are as below;

$$
\begin{aligned}
P & =\left[\begin{array}{ccc}
1 & 0 & 0 \\
0.001 & 0.24975 & 0.74925 \\
0.001 & 0.24975 & 0.74925
\end{array}\right] \\
P^{2} & =\left[\begin{array}{ccc}
1 & 0 & 0 \\
0.002 & 0.2495 & 0.7485 \\
0.002 & 0.2495 & 0.7485
\end{array}\right] \\
P^{3} & =\left[\begin{array}{ccc}
1 & 0 & 0 \\
0.004 & 0.249 & 0.747 \\
0.004 & 0.249 & 0.747
\end{array}\right]
\end{aligned}
$$

Some of paths and probability calculations are as follow;

$$
\begin{gathered}
P\left(X_{2}=0, X_{4}=0\right)=P\left(X_{2}=0\right) P\left(X_{4}-X_{2}=0\right)=P\left(X_{2}=0\right) P\left(X_{2}=0\right) \\
=P\left(X_{2}=0\right)^{2}=\left(P\left(X_{1}=-1\right), P\left(X_{2}=0\right)+P\left(X_{1}=1\right), P\left(X_{2}=0\right)\right)^{2} \\
=\left(P\left(X_{1}=-1\right), P\left(X_{2}=0\right)+P\left(X_{1}=1\right), P\left(X_{2}=0\right)\right)^{2} \\
=\left(P\left(X_{1}=-1\right) P\left(X_{1}=1\right)+P\left(X_{1}=1\right) P\left(X_{1}=-1\right)\right)^{2} \\
=(0.74925 * 0.24975+0.24975 * 0.74925)^{2}=0.14 \\
P\left(X_{4}=2, X_{10}=6\right)=P\left(X_{4}=2\right) P\left(X_{10}-X_{4}=4\right)=P\left(X_{4}=2\right) P\left(X_{6}=4\right) \\
=4 P\left(X_{1}=-1\right) P\left(X_{1}=1\right)^{3} 6 P\left(X_{1}=-1\right) P\left(X_{1}=1\right)^{5}=0.0155 * 0.0043 \\
=0.0198
\end{gathered}
$$

The probabilities of each situation with including every path is in Table 1 .

$$
\begin{gathered}
E\left(X_{n}\right)=\sum_{i=1}^{n} P\left(X_{i}=x_{i}\right) x_{i} \\
E\left(X_{15}\right)=P\left(X_{1}=1\right)^{15} P\left(X_{1}=-1\right)^{0} *(80929 *(1+15 * 0.05-0 * 0.005)) \\
+P\left(X_{1}=1\right)^{14} P\left(X_{1}=-1\right)^{1} *(80929 *(1+14 * 0.05-1 * 0.005)) \\
\cdot \\
\cdot \\
\cdot \\
+P\left(X_{1}=1\right)^{0} P\left(X_{1}=-1\right)^{15} *(80929 *(1+0 * 0.05-15 * 0.005)) \\
=49827
\end{gathered}
$$


But in this step we try to predict the conclusion of this pandemic disease. When we continue with the same transition probabilities, we reach a conclusion that this pandemic disease starts to decrease sharply after 23 days in step 5. After 25 days the expectation is 29598, after 32 days the expectation is 15675 . According to expectation of this stochastic process this pandemic is totally wipe out after 36 days.

\section{CONCLUSION}

Related with corona virus we introduce a stochastic approach to this disease. At first we defined the rules of this pandemics' spread. Later we make a study to recognize the spread of this disease step by step. In the end we try to define the approximate time of vanishing for pandemic according to rules of our stochastic process. And according to stochastic process for this system, we conclude that obeying rules is very important to strict spreading of this pandemic. We conclude that if reliable data sets use in the process for defining probabilities in transition matrices, more exact and reliable conclusions will be reached. 


\section{APPENDIX}

\begin{tabular}{|l|l|l|l|l|l|}
\hline $\begin{array}{l}\text { number of } \\
\text { increase }\end{array}$ & STEP 1 & STEP 2 & STEP 3 & STEP 4 & STEP 5 \\
\hline $\mathbf{0}$ & 0,013164 & $3,01 \mathrm{E}-05$ & $9,17 \mathrm{E}-10$ & $3,01 \mathrm{E}-05$ & $9,17 \mathrm{E}-10$ \\
\hline $\mathbf{1}$ & 0,065822 & 0,000451 & $4,13 \mathrm{E}-08$ & 0,000451 & $4,13 \mathrm{E}-08$ \\
\hline $\mathbf{2}$ & 0,153585 & 0,003157 & $8,67 \mathrm{E}-07$ & 0,003157 & $8,67 \mathrm{E}-07$ \\
\hline $\mathbf{3}$ & 0,221845 & 0,013679 & $1,13 \mathrm{E}-05$ & 0,013679 & $1,13 \mathrm{E}-05$ \\
\hline $\mathbf{4}$ & 0,221845 & 0,041036 & 0,000101 & 0,041036 & 0,000101 \\
\hline $\mathbf{5}$ & 0,162686 & 0,090279 & 0,000669 & 0,090279 & 0,000669 \\
\hline $\mathbf{6}$ & 0,090381 & 0,150465 & 0,003347 & 0,150465 & 0,003347 \\
\hline $\mathbf{7}$ & 0,038735 & 0,193455 & 0,012912 & 0,193455 & 0,012912 \\
\hline $\mathbf{8}$ & 0,012912 & 0,193455 & 0,038735 & 0,193455 & 0,038735 \\
\hline $\mathbf{9}$ & 0,003347 & 0,150465 & 0,090381 & 0,150465 & 0,090381 \\
\hline $\mathbf{1 0}$ & 0,000669 & 0,090279 & 0,162686 & 0,090279 & 0,162686 \\
\hline $\mathbf{1 1}$ & 0,000101 & 0,041036 & 0,221845 & 0,041036 & 0,221845 \\
\hline $\mathbf{1 2}$ & $1,13 \mathrm{E}-05$ & 0,013679 & 0,221845 & 0,013679 & 0,221845 \\
\hline $\mathbf{1 3}$ & $8,67 \mathrm{E}-07$ & 0,003157 & 0,153585 & 0,003157 & 0,153585 \\
\hline $\mathbf{1 4}$ & $4,13 \mathrm{E}-08$ & 0,000451 & 0,065822 & 0,000451 & 0,065822 \\
\hline $\mathbf{1 5}$ & $9,17 \mathrm{E}-10$ & $3,01 \mathrm{E}-05$ & 0,013164 & $3,01 \mathrm{E}-05$ & 0,013164 \\
\hline
\end{tabular}

Fig. 2. Probabilities of situations included path combinations. 


\section{REFERENCES}

Ery1lmaz S. 2017. A new class of bivariate lifetime distributions. Communications in StatisticsTheory and Methods. Vol. 46(24), 12324-12335.

Marshall A.W., Olkin I. 1997. A new method of adding a parameter to a family of distributions with application to the exponential and Weibull families, Biometrika 84 641-652

Li X., Zuo M.J. 2004. Preservation of stochastic orders for random minima and maxima with applications, Nav. Res. Logist., 51, 332-344.

Ahmad I., Kayid M. 2007. Reversed preservation of stochastic orders for random minima and maxima with applications, Statistical Papers, 48, 283-293.

Kundu D., Gupta R.D. 2014.On bivariate Weibull-geometric distribution, Journal of Multivariate Analysis, 123, 19-29.

Miaomiao Y., Yinghui T., Wenqing W., Jie Z. 2014. Optimal order-replacement policy for a phase-type geometric process model with extreme shocks. Applied Mathematical Modelling. Vol: $38.4323-4332$

Haijun L. 2003. Association of multivariate phase-type distributions, with applications to shock models. Statistics \& Probability Letters Vol:64, 381-392.

Mai J.F., Scherer M. and Shenkman N. 2013. Multivariate geometric distributions, (logarithmically) monotone sequences, and infinitely divisible laws. Journal of Multivariate Analysis 115 457-480.

Arnold B.C. 1975. A characterization of the exponential distribution by multivariate geometric compounding, Sankhy ${ }^{-}$: The Indian Journal of Statistics 37 (1) 164-173.

Marshall A.W., Olkin I. 1967. A multivariate exponential distribution, Journal of the American Statistical Association 62 (317) 30-44.

Marshall A.W., Olkin I. 1995. Multivariate exponential and geometric distributions with limited memory, Journal of Multivariate Analysis 53 110-125.

Nair N.U., Asha G. 1997. Some classes of multivariate life distributions in discrete time, Journal of Multivariate Analysis 62 181-189.

Jayakumar K. and Mundassery D.A. 2007. On Bivariate Geometric Distribution. Statistica. Vol. 67 (4). 389-404.

Krishna H. and Singh P. 2009. A Bivariate Geometric Distribution with Applications to Reliability.

Klein, M. 1962. Inspection-maintenance-replacement schedules under Markovian deterioration. Management Science, V. 9, No. 1, pp. 25-32.

Barlow R.E. and Proschan F. 1965. Mathematical theory of reliability. John Wiley\& Sons, Inc., New York, NY, 1965. 
Hüseyin Ünözkan,

Department of Statistics, Ankara University

Dögol Cad. Cankaya ANKARA TURKEY.

email: hunozkan@gmail.com

ORCID ID: http://orcid.org/0000-0001-9659-287X

Mehmet Yılmaz,

Department of Statistics, Ankara University

Dögol Cad. Cankaya ANKARA TURKEY.

email: scimehmet@gmail.com

ORCID ID: http://orcid.org/0000-0002-9762-6688

\author{
Ahmet Murat Dere, \\ Department of Industrial Engineering, Gazi University \\ Maltepe ANKARA TURKEY \\ email: ahmet.murat.dere@gazi.edu.tr
}

